\title{
Correlation between microbial host factors and caries among older adults
}

\author{
Rakhi Mittal*iD, Kai Soo Tan, Mun Loke Wong and Patrick Finbarr Allen
}

\begin{abstract}
Background: There is little knowledge about factors which may affect oral health among older adults. The objective of this study was to determine the relationship between Streptococcus mutans (MS) and Lactobacilli (LB) counts and caries among older adults.

Methods: In this community-based observation study, 141 participants aged 60 years and above were recruited from the west district of Singapore. Alongside the clinical examination, saliva samples were collected to determine Streptococcus mutans (MS) and Lactobacilli (LB) counts, as well as to record salivary flow rate and buffering capacity of saliva.

Results: Of the 141 participants, $63.8 \%$ were female and $94.3 \%$ were of Chinese ethnicity. The mean DMFT was 11.08 (s.d. 8.27). 9.9\% of participants had at least one decayed tooth, $52.5 \%$ had minimum one missing tooth and $86.5 \%$ had at least one filled tooth. $67.4 \%$ had MS counts of $\geq 10^{5}$ while LB counts were $\geq 10^{5}$ for $48.2 \% .83 .7 \%$ had normal salivary flow or hypersalivation ( $>=1 \mathrm{~mL} / \mathrm{min}$ ), the buffering capacity of the saliva was alkaline in $61 \%$ of the participants. Multivariate analysis showed that participants who had high MS counts were less likely to have a DMFT < 12 [OR (95\% $\mathrm{Cl}), 0.29(0.11-0.77)]$ whereas participants who had high LB counts were less likely to have a DMFT $\leq 14$ [OR ( $95 \% \mathrm{Cl}$ ), $0.45(0.20-1.002)]$.
\end{abstract}

Conclusion: Our study showed a positive correlation between MS and LB counts and caries experience in older adults. The mean DMFT was on the low side in our sample despite having a relatively high MS count. This suggests that there are many other factors which vary according to host environment, physiological and biological conditions that may affect MS and LB counts in the oral cavity.

Clinical relevance: Our study supports the knowledge that the aetiology of dental caries among older adults is a complex process and it would be wrong to consider caries as a same problem with the same solution for all age groups.

Keywords: Streptococcus, Lactobacilli, Saliva flow, Buffering capacity, Dental caries

\section{Background}

Population growth and aging has led to a dramatic increase in the burden of untreated oral conditions throughout the world in older adults [1]. Untreated cavitated dentine carious lesions in permanent teeth remained the most prevalent health condition across

*Correspondence: drrakhimittal@yahoo.com

Faculty of Dentistry, National University of Singapore, Singapore, Singapore the globe in 2010, affecting 2.4 billion people [2]. The direct and indirect global economic impact of oral conditions may amount to more than US $\$ 442$ billion [3]. Currently, older adults are found to have more of their natural teeth [4] which in turn puts them at greater risk of dental decay. Poor oral health condition of older adults can increase the risk to general health with compromised chewing and eating abilities [5]. Currently, ageing research primarily focuses on managing longterm conditions and improving disability and frailty in 
older age; there is little knowledge about factors which may prevent oral health problems in later life [6].

Dental caries is a multi-factorial, behaviourally mediated infectious disease that involves complex interactions between acid-producing bacteria, fermentable carbohydrates and many other host factors. The aetiology of dental caries involves a myriad of components which differs with age due to physiological changes in the human body [7]. Streptococcus mutans (MS) is one of the well-established etiologic agents for caries in both healthy and medically compromised individuals [8]. The predictive value of salivary levels of MS has been evaluated in many studies; however, the results are not consistent. Although some studies found a significant association between salivary levels of MS and subsequent caries onset [9] other studies revealed no clear-cut association between them $[10,11]$. It has been shown that levels of salivary MS might not be the sole predictor for development of caries [12].

Some studies have indicated a relationship between Lactobacilli (LB) and dental caries [13, 14] whilst others found that caries lesions can develop in the absence of LB $[15,16]$. The relationship between LB and dental caries, at least in humans, has not been proven to be cause-and-effect [16]. Various studies attempted to correlate changes in the composition of oral microflora with age; however there were certain limitations with the design of such studies including: the definition of older adults, their health status and, their level of dependency (housebound or institutionalised) [17].

Little is known about the microbial aetiology of dental caries in older adults (i.e., over 65 years of age) and there is no consensus as to which microbes cause oral diseases [18]. Thus, the predictive value of MS and LB counts in the saliva of older adults resulting in oral diseases remains unclear. Apart from increased colonisation with cariogenic bacterial species in the oral cavity, low salivary flow has also been linked to caries incidence [19]. Normal salivary flow protects the oral cavity, the upper airway and digestive tract and facilitates numerous sensorimotor phenomena [20]. With age, saliva flow rate reduces in older adults, and this may be influenced by medications and pathological changes in the salivary glands [21]. The main attributes of reduced salivary flow is self-reported mouth dryness ("xerostomia") and increased incident dental caries.

Furthermore, few studies have demonstrated strong association between low caries levels and high salivary buffering capacity [22]. In the literature, larger quantities and faster rates of bacterial acid production have been consistently reported in caries active individuals than in caries-free individuals [7].
The influence of salivary flow and $\mathrm{pH}$ on dental caries were evaluated in children, adolescents and adults, whereas studies about the polymicrobial aetiology of dental caries in the older adults are incomplete, and the results remain controversial [23]. There is a little evidence available from epidemiological studies, and most studies lack statistical power [24]. Given the reducing prevalence of tooth loss with associated rise in carious teeth in an aging population, it is important to increase our understanding of the factors which moderate dental caries in older adults. The aim of our research is to assess the relationship between MS and LB levels, saliva flow rate, buffering capacity and coronal caries experience in older adults. The objective was to determine the association between microbial host factors and coronal and root caries in older adults.

\section{Methods \\ Population and sample}

A total of 141 community dwelling older adults were recruited from a senior activity centre located in the central west district of Singapore. The chosen sample for our study is adequate to investigate the objectives of the study. This study was part of Community Health Intergenerational (CHI) study [25]: an observational cohort study on ageing and mental health in community-dwelling older adults.

\section{Patient and public involvement}

Participants in this study were not involved in the development of the study design or objectives. The research design and objectives were developed by the Principal Investigator (PI) and Co-Investigators (Co-Is) of this study and underwent review by a board of academic advisors affiliated to National University of Singapore MindScience Centre (NUS MSC). Presbyterian Community Services (PCS), a community partner, provided the study site (e.g., quiet rooms in Hannah Seniors' Activity Centre (HSAC) to collect data). In addition, information about the study design and recruitment processes were shared with staff of PCS prior to data collection.

\section{Recruitment}

Older adults 60 years and above were recruited from residences in the Toh Yi, Anak Bukit area in Singapore, and other areas within the district encompassed by a $10 \mathrm{~km}$ radius from the HSAC via door-to-door visits by research nurses and research assistants. Eligible individuals were also recruited onsite from HSAC, community centres, resident corners, senior activity centres and residences within the recruitment area-advertisement flyers were made available for visitors to the respective centres and by word of mouth. Interested individuals were invited to 
HSAC at their convenience. There were no specific exclusion criteria, except that participants should be between 60 and 99 years of age. Non-ambulant individuals who were keen to participate in the study gave their consent in their own homes. A member of the research team explained the study in detail and time was given to each individual to consider their participation in the study before signing the consent form.

\section{Dental clinical examination}

The dental clinical examination was carried out by trained dentist in a quiet room located in HSAC. Clinical examination of all participants was carried out on a mobile dental chair. The dental examination included recording details of: (1) coronal status (decay, filled, missing due to decay or other reasons etc.) and root status (decay, filled, missing due to decay or other reasons etc.).

\section{Bacteriological examination}

Alongside the clinical examination of the subject's dentition, saliva samples were also collected to determine MS and LB counts of the subjects, as well as to record salivary flow rate and buffering capacity of saliva. Generally, the determination of both MS and LB count increases accuracy of diagnosis and improves subsequent prognosis in the management of dental caries. Subjects were instructed to chew on paraffin wax for five minutes and spit out their stimulated residual saliva into a sterilised container. The $\mathrm{pH}$ of saliva was tested with a $\mathrm{pH}$ paper to determine its $\mathrm{pH}$ value against a calibrated chart. Secondly, the volume of stimulated saliva was recorded and the salivary flow per minute was subsequently calculated. After which, the saliva was applied onto Ivoclar Vivadent Inc ${ }^{\circledR}$ CRT kit (sneezing or coughing near the agar was avoided for the sake of test stability and mould prevention). Saliva was then allowed to flow on both surfaces of the CRT kit using a pipette without scratching the culture media. Thorough wetting was achieved by holding the carrier slightly oblique to prevent quick flow of saliva, before placing the agar immediately back into the test vial. A sodium bicarbonate tablet was added in the test vial, releasing carbon dioxide upon contact with moisture once the vial was tightly secured. The CRT kit was incubated at $37^{\circ} \mathrm{C}$ for $48 \mathrm{~h}$. The amounts of MS and LB were determined to be more than or less than $10^{5} \mathrm{col}-$ ony forming units (CFU) using a chart provided by the manufacturer.

\section{Other data}

Data on potential covariates were obtained from in-person interviews and included age, gender, ethnicity, education and monthly household income.

\section{Statistical analysis}

The main outcome of this study was DMFT of older adults which was categorised as DMFT. Mean DMFT of coronal caries was 12 and mean DMFT for root caries was 14 . To identify correlation between DMFT and other host factors including: age, gender, ethnicity, education, household income level, volume of saliva, buffering capacity of saliva, MS count and LB count, we first performed bivariate analysis. After bivariate analysis, we conducted multivariate analysis to predict potential factors associated with DMFT of study participants. $P$-value $<0.05$ was considered significant. All statistical analyses were performed using SPSS version 24 .

\section{Results}

\section{Descriptive statistics}

Table 1, shows that the majority of participants were between 60 and 75 years old, with a larger proportion of females (63.8\%). The majority of the participants were of Chinese ethnicity (94.3\%), 53.9\% with monwthly household income of below $\$ \$ 5,000$. The majority of the participants had at least a Primary education level (92.9\%), with $31.9 \%$ having higher level or University level education.

Table 2, shows data related to DMFT index of study population. The mean coronal DMFT was calculated to be 11.08 (Standard Deviation: 8.27). DMFT more than 12 was considered as high and DMFT less than 12 was considered low in this study. $9.9 \%$ of participants had at least one decayed tooth (DT), 52.5\% had minimally one missing tooth (MT) and $86.5 \%$ had at least one filled tooth (FT). In total $34 \%$ of the study sample, had DMFT greater than 12 and the rest had DMFT less than 12. The mean

Table 1 Shows socio-demographic characteristics of study population $(\mathrm{N}=141)$

\begin{tabular}{lr}
\hline Participants characteristics & N (\%) \\
\hline Age & $112(79.4)$ \\
$60-75$ years old & $29(20.6)$ \\
More than 75 years & \\
Gender & $51(36.2)$ \\
Male & $90(63.8)$ \\
Female & \\
Ethnicity & $133(94.3)$ \\
Chinese & $8(5.7)$ \\
Non-Chinese & \\
Monthly household income & $76(53.9)$ \\
Below \$5000 & $65(46.1)$ \\
Above \$5000 & \\
Education level & $10(7.1)$ \\
No formal education & $131(92.9)$ \\
Primary passed and above &
\end{tabular}


Table 2 Shows details about oral health characteristics of study population $(\mathrm{N}=141)$

\begin{tabular}{lc}
\hline Oral health characteristics & $\mathbf{N}(\%)$ \\
\hline Coronal DMFT & \\
DMFT $<12$ & $93(66.0)$ \\
DMFT $\geq 12$ & $48(34.0)$ \\
Decayed & $14(9.9)$ \\
Missing & $74(52.5)$ \\
Filled & $122(86.5)$ \\
Root DMFT & \\
DMFT $\leq 14$ & $90(63.8)$ \\
DMFT $>14$ & $51(36.2)$ \\
Decayed & $18(12.8)$ \\
Missing & $135(95.7)$ \\
Filled & $92(65.2)$ \\
MS count & \\
High $\left(\geq 10^{5}\right)$ & $95(67.4)$ \\
Low $\left(<10^{5}\right)$ & $46(32.6)$ \\
LB count & \\
High $\left(\geq 10^{5}\right)$ & $68(48.2)$ \\
Low $\left(<10^{5}\right)$ & $73(51.8)$ \\
Saliva volume & \\
Normal/hyper-saliva ( $>=1 \mathrm{~mL} / \mathrm{min}$ of saliva) & $118(83.7)$ \\
Hypo-saliva $(<1 \mathrm{~mL} / \mathrm{min}$ of saliva) & $23(16.3)$ \\
Saliva buffering capacity & $15(10.6)$ \\
Acidic & $40(28.4)$ \\
Neutral & $86(61.0)$ \\
Alkaline & \\
\hline
\end{tabular}

*Mean DMFT of study population was 12

root DMFT was calculated to be 13.8 (Standard Deviation: 0.48$) .12 .8 \%$ of participants had at least one decayed tooth (DT), 95.7\% had minimally one missing tooth (MT) and $65.2 \%$ had at least one filled tooth (FT).

For bacterial count, $67.4 \%$ had high MS count of $\geq 10^{5}$ while $32.6 \%$ had $<10^{5}$ MS count. LB count was $\geq 10^{5}$ for $48.2 \%$ of the study sample, while $51.8 \%$ of the participants had $<10^{5}$ LB count.

Our results showed that $83.7 \%$ of participants had normal salivary flow or hypersalivation $(>=1 \mathrm{~mL} / \mathrm{min})$ while $16.3 \%$ had hypo salivation $(<1 \mathrm{~mL} / \mathrm{min}$ of saliva). Buffering capacity of the saliva was mostly alkaline for the majority of the participants (61\%), while $28.4 \%$ of them had saliva which was neutral and only $10.6 \%$ of them had acidic saliva.

\section{Correlates of coronal caries in the study population Bivariate analysis}

Table 3 shows bivariate correlation between DMFT and characteristics of study population. Significant correlation was found between coronal DMFT and MS count
Table 3 Shows bivariate correlation between DMFT and characteristics of study population $(\mathrm{N}=141)$

\begin{tabular}{llc}
\hline Study population characteristics & $\begin{array}{l}\text { Coronal caries } \\
\text { Bivariate } \\
\text { analysis } \\
\text { ( } \boldsymbol{p} \text {-value) }\end{array}$ & $\begin{array}{l}\text { Root caries } \\
\text { Bivariate } \\
\text { analysis } \\
\text { ( } \boldsymbol{p} \text {-value) }\end{array}$ \\
\hline Age & 0.40 & 0.12 \\
Gender & 0.52 & 0.10 \\
Ethnicity & 0.17 & -0.12 \\
Education & 0.08 & -0.13 \\
Household income & 0.36 & -0.16 \\
MS count & $0.002^{\mathrm{a}}$ & 0.17 \\
LB count & $0.009^{\mathrm{a}}$ & $<0.01^{\mathrm{a}}$ \\
Saliva volume & 0.15 & 0.96 \\
Saliva buffering capacity & 0.34 & 0.23
\end{tabular}

a Significant $(p<0.05)$

$(p=0.002)$ and LB count $(p=0.009)$. However, DMFT was not found significantly correlated with salivary volume $(p=0.15)$ and buffering capacity $(p=0.34)$. Root DMFT was found significantly correlated with LB count $(\mathrm{p}<0.01)$.

\section{Multivariate analysis}

Next, we performed multivariate analysis to predict potential host factors associated with coronal and root DMFT. After adjusting for covariates, we found that study participants who had high MS count were less likely to have a coronal DMFT $<12$ [OR (95\% CI), 0.29 (0.11-0.77)] whereas study participants who had high LB count were less likely to have root DMFT $\leq 14$ (Table 4).

\section{Discussion}

To our knowledge, this is the first study of dental caries conducted within a community-based research network in Singapore. The findings of our study emphasise that aetiology of dental caries is a complex process and it would be wrong to consider caries as a same problem with the same solution for all age groups [26]. Oral microbiome changes along with ageing [13] and many aspects of cultural diversity can influence oral hygiene routines, diet, health beliefs, and access to care, which may in turn affect oral health status [27].

Our study population had low DT scores and still MS count was found to be significantly associated with DMFT score. MS is considered to be a specific microorganism that causes dental caries [7], there is evidence where MS count was low in presence of caries [28, 29]. In the present study, DT score is very low compared to MT and FT score, with majority of the study population having at least one filled tooth. For several years, amalgam 
Table 4 Shows multivariate analysis between DMFT and host characteristics

\begin{tabular}{|c|c|c|c|c|}
\hline \multirow[t]{3}{*}{ Participants characteristics } & \multicolumn{2}{|l|}{ Coronal caries } & \multicolumn{2}{|l|}{ Root caries } \\
\hline & Multivariate analysis & $p$ value & Multivariate analysis & $p$ value \\
\hline & OR (95\% Cl) & & OR $(95 \% \mathrm{Cl})$ & \\
\hline \multicolumn{5}{|l|}{ Age } \\
\hline $60-75$ years & $1.15(0.42-3.15)$ & 0.77 & $1.22(0.46-3.19)$ & 0.68 \\
\hline More than 75 years & - & & - & \\
\hline \multicolumn{5}{|l|}{ Gender } \\
\hline Male & $1.11(0.49-2.52)$ & 0.78 & $1.55(0.69-3.50)$ & 0.28 \\
\hline Female & - & & - & \\
\hline \multicolumn{5}{|l|}{ Ethnicity } \\
\hline Chinese & $0.19(0.02-1.83)$ & 0.15 & $0.14(0.01-1.44)$ & 0.10 \\
\hline Non-Chinese & - & & - & \\
\hline \multicolumn{5}{|l|}{ Education } \\
\hline No formal education & $0.57(0.11-2.80)$ & 0.49 & $0.44(0.08-2.26)$ & 0.32 \\
\hline Primary and above & - & & - & \\
\hline \multicolumn{5}{|l|}{ Household income } \\
\hline Below $\$ 5,000$ & $0.69(0.30-1.54)$ & 0.36 & $0.54(0.24-1.19)$ & 0.12 \\
\hline$>=\$ 5,000$ & - & & - & \\
\hline \multicolumn{5}{|l|}{ Volume of saliva } \\
\hline Normal/hyper (>=5 ml) & $1.51(0.47-4.78)$ & 0.48 & $0.45(0.13-1.56)$ & 0.21 \\
\hline Hypo (<5 ml) & - & & - & \\
\hline \multicolumn{5}{|l|}{ Buffering capacity } \\
\hline Alkaline $(\mathrm{pH}>7)$ & $1.13(0.28-4.45)$ & 0.85 & $1.70(0.45-6.42)$ & 0.42 \\
\hline Neutral $(\mathrm{pH}=7)$ & $1.11(0.27-4.56)$ & 0.88 & $1.42(0.35-5.63)$ & 0.61 \\
\hline Acidic $(\mathrm{pH}<7)$ & - & & - & \\
\hline \multicolumn{5}{|l|}{ MS count } \\
\hline $\operatorname{High}\left(>=10^{5}\right)$ & $0.29(0.11-0.77)$ & $0.01^{*}$ & $0.67(0.27-1.62)$ & 0.37 \\
\hline $\operatorname{Low}\left(<10^{5}\right)$ & - & & - & \\
\hline \multicolumn{5}{|l|}{ LB count } \\
\hline $\operatorname{High}\left(>=10^{5}\right)$ & $0.58(0.26-1.29)$ & 0.18 & $0.45(0.20-1.002)$ & $0.05^{*}$ \\
\hline $\operatorname{Low}\left(<10^{5}\right)$ & - & & - & \\
\hline
\end{tabular}

Outcome: DMFT, reference group: DMFT $\geq 12 ;{ }^{*}$ Significant $(p<0.05)$

filling has been considered as the main restorative material specifically for posterior teeth, however, at present all individuals prefer resin composite restorations because of their good aesthetics and adhesion properties [30]. It was been shown that MS colony forming unit (CFU) from resin composite group were significantly greater than amalgam restoration which means that physical and chemical characteristics of the restorative materials may affect MS count [31]. This validates the finding that MS count in oral cavity depends on various factors and not just on the presence of dental caries. Unfortunately, we didn't record the type of restoration done in our study sample. It will be interesting to correlate the type of restorations with MS and LB counts in future studies.

Our study showed a significant association between root caries and LB count. This relationship should be interpreted with caution. The codes used for recording root status of study population did not include 'missing due to caries' which means that teeth which were missing were considered as missing whereas codes used for recording coronal status included 'missing due to caries' meaning teeth which were missing due to caries were considered as missing. Relationship between LB count and caries is still debatable, future studies should be done to explore and compare association between microbial host factors and coronal and root caries.

Bacteria in dental biofilms are regarded as important in initiation and progression of dental caries [32]. Previous studies showed association between dental caries and bacterial colonies in children, adolescents and adults, but studies about the polymicrobial aetiology of dental caries in older adults are incomplete or the results remain inconsistent [13]. In our study, LB count was found to be significantly associated with coronal caries experience in 
univariate testing, but it did not emerge as a significant variable in the multivariate testing or the final prediction model. At present, general opinion supports the concept that LB is not involved in the initiation of dental caries but more in the progression of deep enamel lesions [33]. Also, it has been reported that LB is not present in adults without active caries lesions [14]. In our study population, there were few active/untreated coronal lesions, and this highlights the need to further investigate the relationship between LB counts and carious activity in a population with higher likelihood of untreated caries, e.g., lower socio economic status (SES). This further emphasises the importance of a longitudinal study design to determine the prevalence of microorganisms in oral cavity of older adults.

It is interesting to note that the present study population showed a shift in DMFT scores from higher number of missing teeth to higher number of filled teeth in older adults. This could possibly be due to their relatively higher SES level, and thus more likely to have lower untreated decay and higher levels on restored teeth. These findings corroborates with previous longitudinal research in which there was a reduction in MT scores compared to FT scores in 65-74 year old senior citizens [34]. Furthermore, it has been proven that DT and MT components increased with decreasing income, while the FT component decreased [35]. The majority of our participants were educated, and significant proportion of participants had monthly household income above $\$ \$ 5,000$. In Singapore, the median monthly household income from work grew by 3.0 per cent in nominal terms from $\$ 9,023$ in 2017 to $\$ 9,293$ in 2018 , or 2.6 per cent in real terms. After accounting for household size, the median monthly household income from work per household member rose by 3.4 per cent in nominal terms from $\$ 2,699$ in 2017 to $\$ 2,792$ in 2018 , or 3.0 per cent in real terms [36]. Hence, it can be inferred that our study population falls within the middle-higher income group.

This study characterised stimulated salivary flow and buffering capacity in a group of older Singaporeans. It has been documented that salivary flow varies according to the different parts of the mouth where it is measured and also between persons and across different biological situations [37]. Majority of our participants had greater than equal to $1 \mathrm{ml} / \mathrm{min}$ of saliva flow with an average of $1.87 \mathrm{ml} / \mathrm{min}$. Our average value of saliva flow is higher than older adults in Japan 60 years old and above which has been reported to be $1.36 \mathrm{ml} / \mathrm{min}$ [38]. Interestingly, majority of our study participants had alkaline buffering capacity. This study used stimulated saliva to determine $\mathrm{pH}$ of saliva. Stimulation of saliva may affect salivary $\mathrm{pH}$ and the concentration of some constituents [39]. Chewing paraffin wax stimulate the parasympathetic response which increases saliva output from the parotid gland [40]. Therefore, stimulated saliva has a high buffering capacity [41], this theory may account for the high buffering capacity in the study population. Future studies may need to evaluate these findings in older adults.

Little is known about other factors apart from physiologic and biological changes responsible for saliva flow and buffering capacity. Salivary flow rate and buffering capacity are influenced by SES [37]. As previously stated, majority of our participants were educated and belonged to middle-higher income group, it has been consistently discussed in literature that individuals with better SES have better health conditions [42].

\section{Conclusion}

This study found that MS and LB are an independent predictor of coronal and root caries respectively in older adults, but no significant association between caries and saliva flow and saliva buffering capacity was noted. Thus, one should interpret with caution an assessment of microbial and host factors to determine caries activity in older adults. Our study provides useful baseline data to inform future studies to look at potential factors which will yield more predictive value in determining caries risk in the older adults. Future longitudinal studies should be undertaken to explore this further, including saliva sample collection at different time points to explore determinants of caries experience in older adults.

\section{Abbreviations}

MS: Streptococcus Mutans; LB: Lactobacilli; CHI: Community Health Intergenerational; PI: Principal investigator; Co-Is: Co-investigators; NUS MSC: National University of Singapore Mind - Science Centre; PCS: Presbyterian Community Services; HSAC: Hannah Seniors Activity Centre; DMFT: Decayed Missing Filled Teeth; CRT: Caries risk test; CFU: Colony forming units; SES: Socio-economic status.

\section{Acknowledgements}

We would like to thank participants, research staff and PI of the study to allow us to collect oral health data of participants.

\section{Authors' contributions}

PF Allen conceived the study (PI of the study). PF Allen, RM, WML and TKS designed the methods. RM collected the data. RM analysed the data and wrote the first draft of the report. All authors interpreted results, commented on drafts and approved the final version of the report.

\section{Funding}

This research project was under the National University of Singapore MindScience Centre (NUS MSC) and was funded by donation grants: (1) Hong Kong and Shanghai Bank Corporation grant for community projects, and (2) Kwan Im Thong Hood Cho Temple for NUS MSC's Dementia Prevention Program.

\section{Availability of data and materials}

The data of this study may not be publicly available due to confidentiality agreements with the participants.

\section{Ethics approval and consent to participate}

Ethics approval for this study was granted by the National University of Singapore-Institutional Review Board (NUS-IRB Reference code: H-17-047) on 12 
October 2017. Written informed consent was obtained from all participants after the objectives and procedures of the research were fully explained to them by a member of the research team. Participants were informed that they could withdraw from the research at any time without giving any reasons. Participants with dementia were asked to invite their legally acceptable representatives to the consent-taking process and data collection. The research team ascertained that the person making a decision on behalf of the participant with dementia, was acting in the best interest of the participant and took into account the participant's concerns and desires.

\section{Consent for publication}

Not applicable.

\section{Competing interests}

The authors declare that they have no known competing financial interests or personal relationships that could have appeared to influence the work reported in this paper.

Received: 1 September 2020 Accepted: 24 January 2021

Published online: 04 February 2021

\section{References}

1. Kassebaum N, Smith A, Bernabé E, Fleming T, Reynolds A, Vos T, Murray C, Marcenes W, Collaborators GOH. Global, regional, and national prevalence, incidence, and disability-adjusted life years for oral conditions for 195 countries, 1990-2015: a systematic analysis for the global burden of diseases, injuries, and risk factors. J Dent Res. 2017;96(4):380-7.

2. Kassebaum N, Bernabé E, Dahiya M, Bhandari B, Murray C, Marcenes W. Global burden of untreated caries: a systematic review and metaregression. J Dent Res. 2015;94(5):650-8.

3. Listl S, Galloway J, Mossey P, Marcenes W. Global economic impact of dental diseases. J Dent Res. 2015;94(10):1355-61.

4. Thomson W. Dental caries experience in older people over time: What can the large cohort studies tell us? Br Dent J. 2004;196(2):89.

5. Walls AW, Steele JG, Sheiham A, Marcenes W, Moynihan PJ. Oral health and nutrition in older people. J Public Health Dent. 2000;60(4):304-7.

6. Ramsay S, Whincup P, Watt R, Tsakos G, Papacosta A, Lennon L, Wannamethee $S$. Burden of poor oral health in older age: findings from a population-based study of older British men. BMJ open. 2015;5(12):e009476.

7. Loesche WJ. Role of Streptococcus mutans in human dental decay. Microbiol Rev. 1986;50(4):353.

8. Hamada S, Slade HD. Biology, immunology, and cariogenicity of Streptococcus mutans. Microbiol Rev. 1980;44(2):331.

9. Thibodeau EA, O'Sullivan DM. Salivary mutans streptococci and caries development in the primary and mixed dentitions of children. Commun Dent Oral Epidemiol. 1999;27(6):406-12.

10. Van Palenstein Helderman WH, Mikx FH, Van'T Hof MA, Truin GJ, Kalsbeek H. The value of salivary bacterial counts as a supplement to past caries experience as caries predictor in children. Eur J Oral Sci. 2001;109(5):312-5.

11. Zhang Q, Bian Z, Fan M, van Palenstein HW. Salivary mutans streptococci counts as indicators in caries risk assessment in 6-7-year-old Chinese children. J Dent. 2007;35(2):177-80.

12. Lenčova E, Broukal Z, Spižek J. Point-of-care salivary microbial tests for detection of cariogenic species — clinical relevance thereof. Folia Microbiol. 2010;55(6):559-68

13. Jiang Q, Liu J, Chen L, Gan N, Yang D. The oral microbiome in the elderly with dental caries and health. Front Cell Infect Microbiol. 2018;8:442.

14. Caufield P, Schön C, Saraithong P, LiY, Argimón S. Oral lactobacilli and dental caries: a model for niche adaptation in humans. J Dent Res. 2015;94(9-suppl):110S-118S.

15. Kleinberg I. A mixed-bacteria ecological approach to understanding the role of the oral bacteria in dental caries causation: an alternative to Streptococcus mutans and the specific-plaque hypothesis. Crit Rev Oral Biol Med. 2002;13(2):108-25.

16. Sims W. Streptococcus mutans and vaccines for dental caries: a personal commentary and critique. Community Dent Health. 1985;2(2):129.

17. Percival RS: Changes in oral microflora and host defences with advanced age. In: Microbiology and Aging. edn.: Berlin: Springer; 2009: 131-152.
18. Ellen R, Banting D, Fillery E. CLINICAL SCIENCE longitudinal microbiological investigation of a hospitalized population of older adults with a high root surface caries risk. J Dent Res. 1985;64(12):1377-81.

19. Pedersen A, Reibel J, Nordgarden H, Bergem H, Jensen J, Nauntofte B. Primary Sjögren's syndrome: salivary gland function and clinical oral findings. Oral Dis. 1999;5(2):128-38.

20. Razak PA, Richard KJ, Thankachan RP, Hafiz KA, Kumar KN, Sameer K. Geriatric oral health: a review article. J Int Oral Health JIOH. 2014;6(6):110.

21. Crossner C-G. Salivary flow rate in children and adolescents. Swed Dent J. 1984;8(6):271-6.

22. Ericsson Y. Clinical investigations of the salivary buffering action. Acta Odontol Scand. 1959;17(2):131-65.

23. Jiang $Q$, Liu J, Chen L, Gan N, Yang D. The oral microbiome in the elderly with dental caries and health. Front Cell Infect Microbiol. 2019;8:442.

24. Cunha-Cruz J, Scott J, Rothen M, Mancl L, Lawhorn T, Brossel K, Berg J. DENTistry NP-bRCiE-b: salivary characteristics and dental caries: evidence from general dental practices. J Am Dent Assoc. 2013;144(5):e31-40.

25. Lee RZY, Yu J, Rawtaer I, Allen PF, Bao Z, Feng L, Feng Q, Lee JK, Lim CT, Ling LH. CHI study: protocol for an observational cohort study on ageing and mental health in community-dwelling older adults. BMJ open. 2020;10(5):e035003.

26. Lagerweij $M$, Van Loveren C. Declining caries trends: are we satisfied? Curr Oral Health Rep. 2015;2(4):212-7.

27. Prowse S, Schroth RJ, Wilson A, Edwards JM, Sarson J, Levi JA, Moffatt ME: Diversity considerations for promoting early childhood oral health: a pilot study. Int J Dentistry 2014, 2014.

28. Van Houte J, Lopman J, Kent R. The predominant cultivable flora of sound and carious human root surfaces. J Dent Res. 1994;73(11):1727-34.

29. Van Houte J, Lopman J, Kent R. The final pH of bacteria comprising the predominant flora on sound and carious human root and enamel surfaces. J Dent Res. 1996;75(4):1008-14.

30. Ferracane JL. Resin composite - state of the art. Dent Mater. 2011;27(1):29-38.

31. Auschill TM, Arweiler NB, Brecx M, Reich E, Sculean A, Netuschil L. The effect of dental restorative materials on dental biofilm. Eur J Oral Sci. 2002;110(1):48-53.

32. Marsh PD. Microbial ecology of dental plaque and its significance in health and disease. Adv Dent Res. 1994;8(2):263-71.

33. Könönen E: Anaerobic Cocci and Anaerobic Gram-Positive Nonsporulating Bacilli. In: Mandell, Douglas, and Bennett's Principles and Practice of Infectious Diseases. edn.: Elsevier; 2015: 2781-2786. e2782.

34. Carvalho JC, Schiffner U. Dental Caries in European Adults and Senior Citizens 1996-2016: ORCA Saturday Afternoon Symposium in Greifswald, Germany-Part II. Caries Res. 2019;53(3):242-52.

35. López R, Smith PC, Göstemeyer G, Schwendicke F. Ageing, dental caries and periodontal diseases. J Clin Periodontol. 2017;44:S145-52.

36. Key Household Income Trends, 2018 [https://www.singstat.gov.sg/-/media/ files/publications/households/pp-s25.pdf]

37. Islas-Granillo H, Borges-Yañez S, Medina-Solís C, Galan-Vidal C, NavarreteHernández J, Escoffié-Ramirez M, Maupome G. Salivary parameters (salivary flow, $\mathrm{pH}$ and buffering capacity) in stimulated saliva of Mexican elders 60 years old and older. West Indian Med J. 2014;63(7):758.

38. Ikebe K, Matsuda K-I, Morii K, Hazeyama T, Kagawa R, Ogawa T, Nokubi T. Relationship between bite force and salivary flow in older adults. Oral Surg Oral Med Oral Pathol Oral Radiol Endodontol. 2007;104(4):510-5.

39. Liu J, Duan Y. Saliva: a potential media for disease diagnostics and monitoring. Oral Oncol. 2012;48(7):569-77.

40. Gittings S, Turnbull N, Henry B, Roberts CJ, Gershkovich P. Characterisation of human saliva as a platform for oral dissolution medium development. Eur J Pharm Biopharm. 2015;91:16-24.

41. Khemiss M, Khelifa MB, Saad HB: Preliminary findings on the correlation of saliva $\mathrm{pH}$, buffering capacity, flow rate and consistency in relation to waterpipe tobacco smoking. Libyan J Med 2017, 12, 1.

42. Kawachi I. Why social epidemiology? Australasian Epidemiologist. 2000;7(3):5.

\section{Publisher's Note}

Springer Nature remains neutral with regard to jurisdictional claims in published maps and institutional affiliations. 